\title{
INVERTEBRADOS CONTINENTALES DE LAS ISLAS COLUMBRETES. NUEVAS ESPECIES
}

\author{
E. Mestre-Forés ${ }^{1}$, S. Montagud ${ }^{2}$, J. A. Jaques ${ }^{3} \&$ P. González ${ }^{4}$ \\ ${ }^{1,3}$ Departament de Ciéncies Agràries i Medi Natural. Universitat Jaume I. Avinguda de Vicent Sos Banyat, s/n, 12017, Castelló \\ de la Plana (Castelló). EMF: https://orcid.org/0000-0003-0278-1325 - JAJ: https://orcid.org/0000-0003-1353-1727 \\ ${ }^{2}$ Museo [UV] Història Natural. Universitat de València. C/. Dr. Moliner, 50, 46100, Burjassot (Valencia). \\ ESPAÑA. https://orcid.org/0000-0002-0634-4643 \\ ${ }^{4}$ Departamento de Ciencias Biomédicas, Facultad de Ciencias de la Salud, Universidad Cardenal Herrera-CEU, CEU \\ Universities, C/ Ramón y Cajal s/n, 46115 Alfara del Patriarca (Valencia). https://orcid.org/0000-0002-6970-1134
}

Corresponding autor: patricia.gonzalez4@uchceu.es

\section{RESUMEN}

La fauna terrestre de invertebrados del archipiélago protegido de Columbretes es poco conocida. Además, los pocos estudios existentes se centran en su isla principal, Illa Grossa. Aquí presentamos el listado de especies de invertebrados no registradas, hasta el momento, en el archipiélago, resultados obtenidos mediante una combinación de capturas con trampas de caída, avistamientos y recolecciones aleatorias realizadas tanto en Illa Grossa como en otros tres islotes de este archipiélago. Mediante el primer método se recolectaron un total de 14.830 especímenes entre 2008-2011. La mayor cantidad de individuos recolectados mediante este método resultaron ser coleópteros tenebriónidos. Los otros dos métodos proporcionaron 514 registros para el período 2004-2013. En total, se han identificado 45 nuevos taxones correspondientes a 40 géneros y 16 familias. La mayoría de las nuevas especies encontradas no fueron frecuentes a lo largo del período de muestreo. Entre ellas, figuran especies típicas de hábitats antrópicos (p.e. Cydnus aterrimus o Gryllus bimaculatus). Sorprendentemente, también se localizaron en ocasiones especies con capacidades de dispersión limitadas y no presentes en islas pequeñas (p.e. Cebrio superbus).

Palabras clave: Islas Columbretes, artrópodos, nuevas citas, trampas de caída.

\section{ABSTRACT}

\section{Land invertebrates of Columbretes Islands. New species}

The terrestrial invertebrate fauna of the protected archipelago of Columbretes is poorly known. Moreover, the few existing studies are centered on its main island, Illa Grossa. Herein we present the list of invertebrate species not cited so far in the archipelago, results of a combination of pitfall trap catches, direct visual observations, and random collections performed in Illa Grossa as well as in three additional islets of this archipelago. The first method resulted in 14.830 specimens collected from 2008-2011. The highest number of individuals collected by this method were tenebrionid beetles. The other two methods provided 514 records for the period 2004-2013. In total, 45 new records corresponding to 40 genera in 16 families were identified. Most of the new species could not be regularly found during the whole sampling period. Some typical of anthropogenic habitats species were found (i.e., Cydnus aterrimus, Gryllus bimaculatus). Remarkably, some species with limited dispersal abilities and not common in small islands (i.e., Cebrio superbus) have been found.

Keywords: Columbretes Islands, arthropods, new data, pitfall traps.

Cómo citar este artículo/Citation: Mestre-Forés, E., Montagud, S., Jaques, J. A. \& González, P. 2020. Invertebrados continentales de las Islas Columbretes. Nuevas especies. Grael/sia, 76(1): e102. https://doi.org/10.3989/graellsia.2020. v76.236

Copyright: (C) 2020 SAM \& CSIC. This is an open-access article distributed under the terms of the Creative Commons Attribution 4.0 International (CC BY 4.0) License. 


\section{Introducción}

En el Mediterráneo occidental, ante las costas de Castellón, España, se sitúan las Islas Columbretes, a $57 \mathrm{~km}$ del cabo de Oropesa y sobre un fondo marino que no llega a los $100 \mathrm{~m}$ de profundidad (Alonso, 1991). Este archipiélago, de 19 hectáreas de superficie está formado por cuatro grupos de pequeñas islas volcánicas (unas 30 en total, entre islotes y escollos) las cuales son la parte visible de un sistema volcánico de extensa superficie, $40 \times 90 \mathrm{~km}$ (Maillard \& Mauffret, 1993).

Su colonización no se produjo hasta mediados del siglo XIX, con la construcción del faro de La Illa Grossa en 1856. A partir de aquel momento, los fareros y sus familias habitaron la isla de manera prácticamente ininterrumpida durante 116 años, a excepción de algunos breves períodos marcados por la Guerra Civil española. Desde el momento de su colonización, la isla con mayor superficie, La Illa Grossa, empieza a sufrir agresiones tales como quemas de vegetación provocadas con el objetivo principal de acabar con las serpientes y los escorpiones (Serrano, 1987), la introducción y suelta de animales domésticos y el uso de los arbustos existentes para leña. Con la automatización del faro, en 1975, las islas quedaron de nuevo deshabitadas. En esos años también sufrió impactos de proyectiles al ser utilizadas como campo de tiro por las fuerzas aéreas española y norteamericana. Estas circunstancias llevaron, finalmente, a la declaración por parte de la Generalitat Valenciana, en 1988, de las Islas Columbretes y su entorno marino como Parque Natural (Decreto 15/88), instalándose los primeros servicios de vigilancia. Desde esta fecha hasta hoy en día, la única población permanente que ha habitado Columbretes son el equipo de guardas, monitores y técnicos. En 1994 el Parque Natural fue recalificado como Reserva Natural por la Ley 11/94 de Espacios Naturales Protegidos de la Comunidad Valenciana. En 1998, la Consejería de Medio Ambiente, declaró dos microreservas vegetales, una en La Ferrera y otra en La Foradada, cada una de poco más de 1,5 ha de superficie. Debido a estas figuras de protección, su reducida dimensión, vulnerabilidad, dificultades logísticas y lejanía respecto a la costa más cercana, los estudios científicos en el archipiélago acometidos por personal investigador, externo a la Reserva, son más escasos y difíciles de acometer que en otras islas del Mediterráneo.

Los datos más recientes encontrados sobre el clima de Columbretes nos indican que este es termo mediteráneo atenuado con una época seca corta, con datos medios de temperatura, precipitación anual acumulada y número de días de lluvia al año de $18.41^{\circ} \mathrm{C}$, 407,52 mm y 54,7 respectivamente (Mestre-Forés, 2017). Entre otros factores, el clima ha influido en la composición de la fauna y la flora de las Columbretes, pero su aislamiento ha sido clave en la aparición de nuevas especies y subespecies endémicas de animales y plantas. Entre ellas podemos encontrar especies vegetales como Medicago citrina (Font Quer) Greuter, 1986 y Lobularia maritima columbretensis R. Fern., 1992, vertebrados como la lagartija Podarcis atrata Boscá, 1916 y, como invertebrados, diversas especies de coleópteros tenebriónidos como Tentyria pazi Español, 1958, Mordellistenula plutonica Compte, 1969 o Glabrasida bonacherai Español, 1958, o el caracol Xerocrassa (Xerocrassa) molinae (Hidalgo, 1883) (Domingo et al., 2007).

Mientras que las aportaciones en materia de ornitología, herpetología o botánica sobre las Islas Columbretes son más abundantes y están actualizadas, en lo referente a la fauna invertebrada terrestre existen lagunas importantes. Esto se pudo comprobar al realizar la búsqueda bibliográfica de antecedentes pues, aunque nos consta que se han intentado realizar catalogaciones en diferentes ocasiones, debido a las peculiares características anteriormente expuestas de este paraje protegido, las aportaciones sobre este tipo de fauna son escasas. En consecuencia, no existía un catálogo actualizado que recogiera información completa y detallada sobre la fauna invertebrada de las islas Columbretes.

El primero de los trabajos que recoge datos sobre invertebrados de Columbretes, fue realizado por el Archiduque Luis Salvador de Habsburgo-Lorena y Borbón, que lo incluyó como parte de su monografía sobre estas islas (Habsburg-Lothringen, 1895). Hasta el trabajo de Español (1958) no se hizo un verdadero compendio de la fauna invertebrada catalogada hasta ese momento en el archipiélago. Con posterioridad, se publicó el trabajo de Compte (1969), que describe nuevas especies de coleópteros y aporta una relación exhaustiva de las especies de este orden colectadas en su expedición. García-Marí et al. (1991) hacen lo propio para el conjunto de artrópodos, así como Jaeckel (1952) y Robles (1991) sobre gasterópodos. La primera aportación al conocimiento de la nematofauna de las islas Columbretes la publicó Gadea (1974) estudiando las muestras de líquenes sobre roca volcánica básica recolectadas durante una expedición botánica por Xavier Llimona en mayo de 1973 a La Illa Grossa, La Ferrera y La Foradada. A este respecto, Navarro et al. (2015) publican un listado de nematodos liquenícolas obtenido de prospecciones y colectas propias.

En cuanto a especies concretas, Bordera (1998) describe el himenóptero icneumónido endémico Fianoniella stenognatha Bordera, 1998, como nueva especie en La Illa Grossa. Castilla et al. (2004, 2005, 2006, 2010a), publicaron diversos estudios ecológicos sobre las arañas Argiope lobata (Forskal, 1775) y A. bruennichi (Scopoli, 1772), y sobre el escorpión Buthus occitanus (Amoreux, 1789) (Castilla, 1993; Castilla \& Pons, 2007; Castilla et al., 2009, 2010b).

El resto de antecedentes bibliográficos localizados son, o bien estudios sobre algún otro aspecto de estas 
islas, pero en el que de forma secundaria se contemplan también los invertebrados de Columbretes, como en Castilla et al. (2008), donde cita a los isópodos Ligia italica Fabricius, 1798 y Armadillo officinalis Duméril, 1816, como parte de la dieta de las lagartijas de Columbretes; o bien son estudios que incluyen a Columbretes, pero dentro de un trabajo geográfico más amplio, como el de Cartagena (2002) y Cartagena y Galante (2002), que realizaron estudios sobre tenebriónidos en el que comparan este enclave con otras Islas del Mediterráneo. Melià (2003), en su estudio faunístico sobre los pulgones de Castellón, cita a Brachyunguis (Xerophilaphis) suaedus (Paik, 1965), encontrada en junio de 1996 en Columbretes, sobre Suaeda vera Forsskal ex J.F. Gmelin. Trabajos sobre endemismos regionales o estatales incluyen los endemismos ya conocidos de Columbretes (Domingo et al., 2007; Martínez-Ortí \& Puente, 2011). Por último, Prieto \& Háva (2013) en sus aportaciones corológicas sobre el género Dermestes Linnaeus, 1758 (Coleoptera: Dermestidae), sitúa a este género también en el archipiélago.

Además de estas publicaciones, existe una tesis doctoral que incluye datos sobre invertebrados de Columbretes, concretamente sobre la biología de los dípteros sírfidos en ecosistemas insulares de la Comunidad Valenciana y dónde se presentan once nuevas citas de dípteros sírfidos y un par de himenópteros ápidos en Columbretes (Pérez-Bañón, 2000).
Entre los años 2004 y 2013, dos de las autoras realizaron distintas estancias en el archipiélago con el objetivo de elaborar diferentes estudios e informes técnicos. Tras los resultados obtenidos de los mismos y las deficiencias encontradas en cuanto a antecedentes, se convino en la necesidad de registrar el listado de especies de invertebrados no citadas hasta el momento en el archipiélago, siendo estos datos los que se muestran en este artículo.

\section{Material y métodos}

\section{Área de estudio}

El archipiélago de Columbretes está compuesto por los siguientes grupos de islas e islotes (Fig. 1):

- Grupo de La Illa Grossa, formado por La Illa Grossa (1) (conocida también como La Isla, Columbrete Grande, Columbrete Mayor, La Grossa o La Herradura, porque forma un arco de unos $1.250 \mathrm{~m}$ de longitud y que llega a los $67,48 \mathrm{~m}$ de altura en su extremo norte, siendo este el punto más alto de todo el archipiélago) y los islotes Mascarat (2), Senyoreta y Mancolibre (3).

- $\quad$ Grupo de La Ferrera (4), formado por La Ferrera (conocida también como Malaespina, con poco más de $43 \mathrm{~m}$ de altura) y tres islotes, Ferrereta (o Bauzà), Valdés y Navarrete.

- Grupo de La Foradada (5), formado por La Foradada (que también recibe el nombre de Horadada o Ferrer,

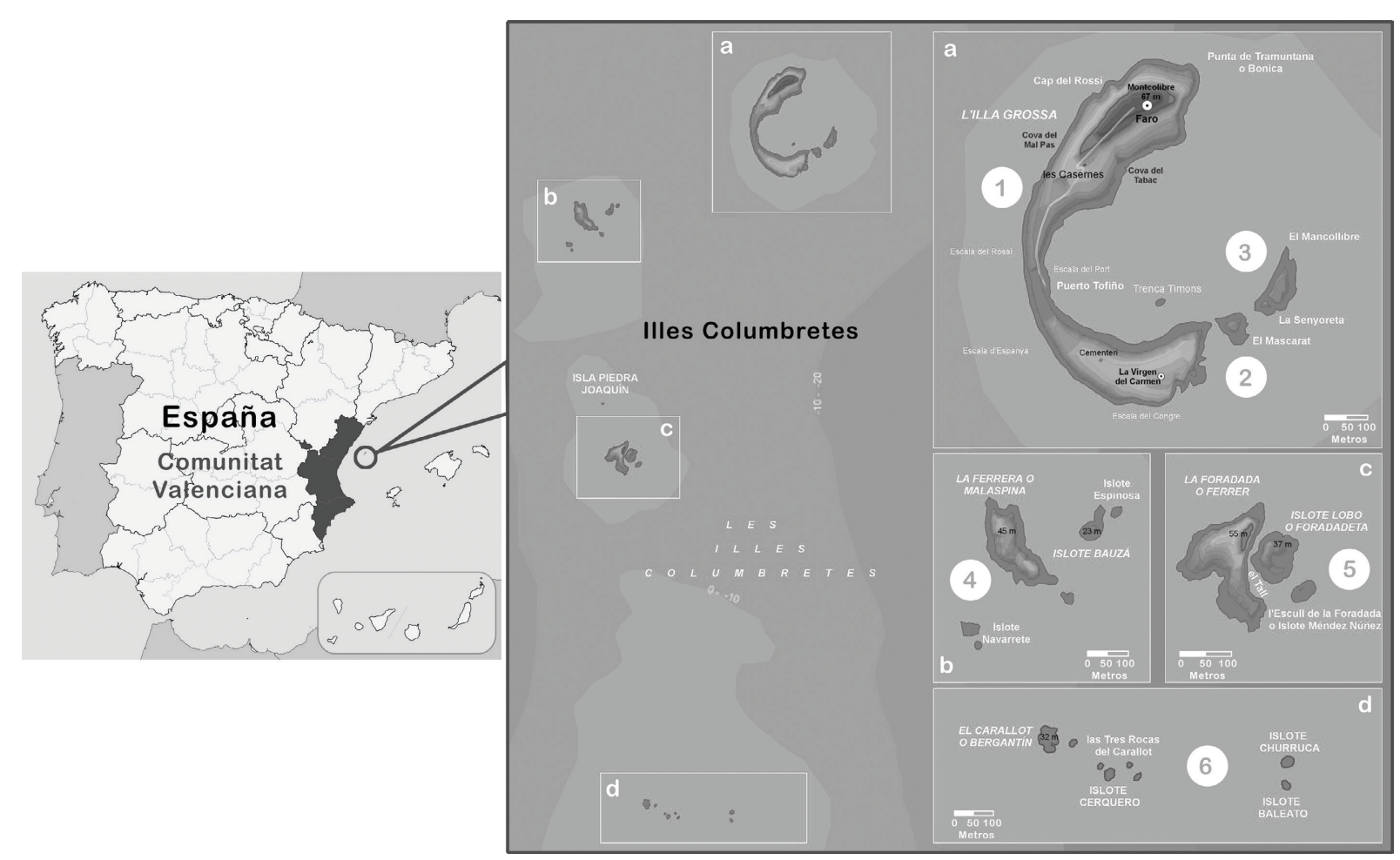

Fig. 1.- Archipiélago de las Islas Columbretes. Los números hacen referencia en el texto a cada uno de los grupos de islotes. Fuente: Elaboración propia a partir de Universitat de València.

Fig. 1.- Columbretes Islands archipelago. The numbers refer in the text to each of the groups of islets. Source: Own elaboration from Universitat de València. 
con una altura de poco más de $55 \mathrm{~m}$ ) y tres islotes, Foradadeta (o Lobo) y Méndez Núñez. Un poco más separado, hacia el norte se localiza Piedra Joaquín.

- Grupo del Carallot (6), formado por El Carallot (que recibe también los nombres de Bergantín o Galiano, con $32 \mathrm{~m}$ de altura) y los islotes Cerquero, Churruca, y Baleato y el escudo Luyando (Smyth, 1831).

El grupo de La Illa Grossa, situado más al norte, aunque es el más grande, cuenta tan solo con 14 hectáreas de superficie. Se formó durante cuatro episodios volcánicos que sucedieron hace entre 300.000 y 1 millón de años (Aparicio et al., 1991) y es el único grupo en que se pueden identificar aún los restos del edificio volcánico (Muñoz et al., 2005). En los otros tres grupos de islotes, las estructuras volcánicas originales prácticamente no se reconocen debido a la erosión marina que han sufrido con posterioridad (Muñoz et al., 2005), presentando acantilados que las hacen casi inaccesibles.

\section{TÉCNICAS DE MUESTREO}

Se han utilizado tres métodos para la captura de los invertebrados: trampas de caída, avistamientos y colectas directas. El muestreo se ha planteado aprovechando la presencia de personal permanente en las Islas, de esta manera se pudo realizar un seguimiento mensual a lo largo de 3 años consecutivos para las trampas de caída y se consiguió recopilar los datos de avistamientos y colectas registrados a lo largo de 9 años.

Se ha incidido en islas e islotes diferentes de La Illa Grossa, a fin de obtener una información global de todo el archipiélago, por lo que incluimos en el planteamiento del estudio los islotes de El Mancolibre, La Ferrera y La Foradada.

\section{Trampas de caída o gravedad}

En La Illa Grossa se colocaron un total de 12 trampas (Southwood \& Henderson, 2000), siguiendo la metodología de trabajos similares en islas mediterráneas (Cartagena \& Galante, 2002). Se tuvo en cuenta el relieve, la presencia humana y la mayor o menor cobertura vegetal a la hora de elegir la disposición de las trampas en los diferentes hábitats de muestreo.

Se utilizaron botes de cristal de $6,6 \mathrm{~cm}$ de diámetro y $11 \mathrm{~cm}$ de profundidad. No se colocó ningún tipo de líquido conservante ni atrayente en su interior, ya que los individuos capturados se volvían a liberar tras su conteo e identificación, siempre a más de cinco metros de las trampas para impedir, en la medida de lo posible, su recaptura. Para dificultar que el brillo del borde del bote resultara atractivo para las gaviotas, animal muy abundante en este archipiélago durante todo el año, se colocó una estructura a modo de "tapa" que permitía la entrada de los artrópodos, pero no el acceso a las aves. Esta tapa también evitaba que durante el día la temperatura en las trampas se elevara demasiado (Fig. 2). En el caso concreto de los tenebriónidos, y con el objetivo de evitar recapturas, se marcaban los individuos recolectados para evitar así duplicaciones en el conteo.

Se realizaron tres ciclos anuales de muestreo, desde agosto de 2008 hasta julio de 2011. Mensualmente, por lo general entre el 10 y el 15 de cada mes (salvo que las circunstancias lo impidieran), se realizó el proceso descrito a continuación:

- Día 1: se colocaban las trampas al anochecer, y se mantenían abiertas y en su lugar durante las siguientes 72 horas.

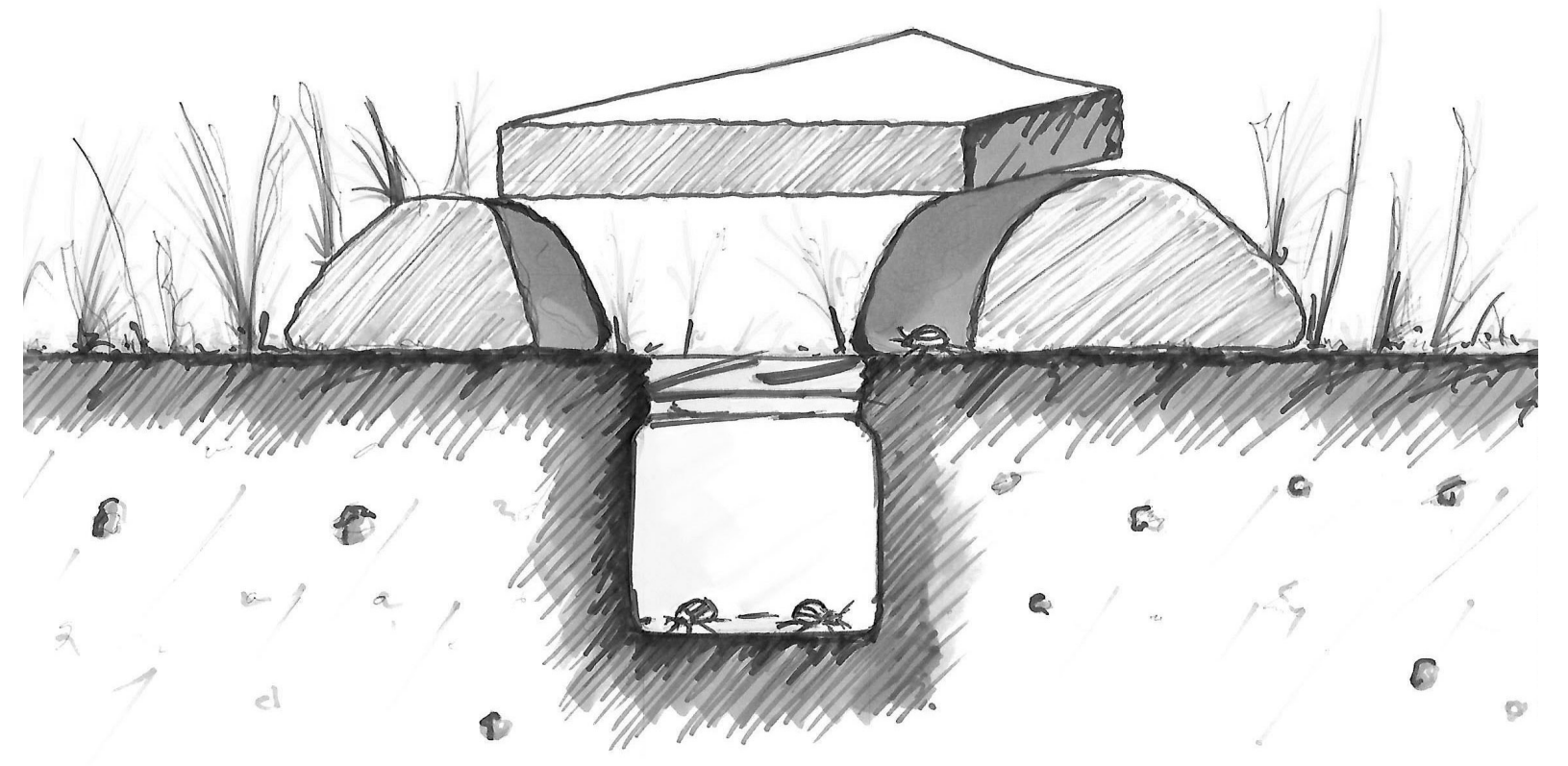

Fig. 2.- Trampa de caída con "tapa". Fuente: elaboración propia.

Fig. 2.- Pitfall trap with "cover". Source: Own elaboration. 
- Días 2, 3 y 4: se realizaban dos visitas diarias, a primera hora de la mañana y al anochecer, en las cuales se contaban ejemplares, se recolectaban y/o fotografiaban especies y se soltaban.

- Tras 72 horas de exposición se recogían las trampas al anochecer.

Aunque somos conscientes que lo más oportuno para este estudio habría sido extender este mismo diseño de muestreo al resto de islotes del archipiélago, por la normativa de la Reserva Natural, cuestiones logísticas, y debido a la dificultad de acceso y su vulnerabilidad, La Ferrera, La Foradada y El Mancolibre se visitaron únicamente de cinco a siete veces a lo largo de los tres años de estudio, colocando las trampas siempre en zona de matorral y tan solo durante doce horas, de manera que se colocaron las trampas antes del anochecer para recogerlas a la mañana siguiente. En La Foradada se colocaron las trampas durante una noche en cada uno de los siguientes meses: junio y julio de 2008, julio de 2009, agosto y septiembre de 2010 y marzo de 2011. En La Ferrera, en los meses: junio y julio de 2008, julio de 2009, mayo, agosto y septiembre de 2010 y marzo de 2011. En El Mancolibre, en los meses de agosto de 2009, mayo, agosto y septiembre de 2010 y febrero de 2011.

En cada visita a las trampas, los artrópodos que aún no estaban identificados se fotografiaban o se conservaban en botes en alcohol de $70^{\circ}$ para su posterior identificación. Si en las trampas de caída se recogían gran cantidad de individuos de una misma especie, estos fueron considerados como un único avistamiento de dicha especie por isla-islote y año a efectos de su catalogación.

Como los registros obtenidos a partir de las trampas de caída solamente proporcionan información sobre los invertebrados no alados, que son los que caen en ellas y no pueden salir (Southwood \& Henderson, 2000), el estudio se ha completado con observaciones directas (avistamientos y colectas).

\section{Avistamientos y colectas}

Complementariamente a las trampas de caída, también se tuvo en cuenta las observaciones puntuales de invertebrados que entre 2004 y 2013 realizaron guardas, monitores y técnicos medioambientales de la Reserva Natural de Columbretes. La mayoría de estas observaciones tuvieron lugar en la zona con mayor presión antrópica de La Illa Grossa, alrededor de "Las Casernas" (nombre tradicional que reciben las viviendas del personal desde el siglo XIX (HabsburgLothringen, 1895).

En todos los casos se fotografiaron y, o bien se conservaron en alcohol $70^{\circ}$ dentro de pequeños frascos debidamente etiquetados (a esto lo denominaremos "colecta"), o bien se identificaron en el momento y se liberaron (es lo que denominaremos "avistamiento"). Al conjunto de avistamientos y colectas lo llamaremos "registro". Los datos de recogida de la muestra y fotografía del individuo se introdujeron en una base de datos que incluía el código de la muestra o imagen, junto con fecha, lugar y comentarios. Las fotografías fueron introducidas en un programa informático debidamente codificadas. Las observaciones realizadas con anterioridad a 2008, de las cuales se disponía de fotografías, fueron introducidas en la misma base de datos siguiendo el mismo procedimiento.

La mayor parte de las muestras colectadas se enviaron para su determinación al personal de la Fundación Entomológica Torres Sala - Museu Valencià d'Història Natural (MVHN). Desde esta institución, se coordinó el envío y cesión de las muestras a determinados especialistas para su identificación.

Para la nomenclatura taxonómica se ha seguido el criterio establecido en Fauna Europaea versión 2.6.2. (www.faunaeur.org). En el caso de las citas identificadas hasta género, se consideran primeras citas si no se había identificado con anterioridad ningún taxón de dicha categoría.

Tras esta metodología se presenta en este trabajo el listado de nuevas citas de invertebrados continentales para el archipiélago de las Islas Columbretes y aquellas que, aunque estaban ya citadas en la Illa Grossa con anterioridad, no se habían registrado todavía en el resto de las islas o islotes muestreados.

\section{Resultados}

A lo largo de los tres años de estudio, en las trampas de caída de La Illa Grossa, se contabilizaron un total de 13.556 invertebrados pertenecientes a 13 órdenes (Tabla 1) mientras que en las de La Ferrera,

\begin{abstract}
Tabla 1.- Número de invertebrados caídos en las trampas de caída de La Illa Grossa a lo largo de 3 años, agrupados por órdenes taxonómicos. Entre paréntesis se indica el porcentaje respecto al total.
\end{abstract}

Table 1.- Number of invertebrates fallen in Illa Grossa pitfall traps over 3 years, grouped by orders. The percentage with respect to the total is indicated in parentheses

\begin{tabular}{llll}
\hline Phylum & Clase & Orden & Total \\
\hline Arthropoda & & Coleoptera & $12.028(88,7 \%)$ \\
& & Hemiptera & $1.028(7,6 \%)$ \\
& & Zygentoma & $38(0,3 \%)$ \\
& Insecta & Diptera & $22(0,2 \%)$ \\
& & Hymenoptera & $15(0,1 \%)$ \\
& & Dictyoptera & $11(0,1 \%)$ \\
& & Orthoptera & $1(<0,1 \%)$ \\
& Malacostraca & Isopoda & $311(2,3 \%)$ \\
& & Scorpiones & $62(0,5 \%)$ \\
Mollusca & Gastropoda & Araneae & $19(0,1 \%)$ \\
Total & & Pulmonata & $2(<0,1 \%)$ \\
\hline
\end{tabular}


Tabla 2.- Número de invertebrados recogidos en las trampas de caída de La Ferrera, La Foradada y El Mancolibre agrupados por órdenes. Entre paréntesis se indica el porcentaje respecto del total en cada isla.

Table 2.- Number of invertebrates fallen in La Ferrera, La Foradada and Mancolibre pitfall traps grouped by orders. The percentage in relation to the total on each island is indicated in parentheses.

\begin{tabular}{lllccc}
\hline Phylum & Clase & Orden & Ferrera & Foradada & Mancolibre \\
\hline Arthropoda & Insecta & Coleoptera & $344(82,1 \%)$ & $366(86,5 \%)$ & $251(58,1 \%)$ \\
& & Hemiptera & 0 & $2(0,5 \%)$ & 0 \\
& & Hymenoptera & 0 & $1(0,2 \%)$ & 0 \\
& & Orthoptera & $3(0,7 \%)$ & 0 & 0 \\
& Malacostraca & Isopoda & $42(10,05)$ & $24(5,7 \%)$ & $178(41,2 \%)$ \\
& Arachnida & Scorpiones & $30(7,2 \%)$ & $28(6,6 \%)$ & $3(0,7 \%)$ \\
Total & & Araneae & 0 & $2(0,5 \%)$ & 0 \\
$\mathbf{N}^{\circ}$ meses de muestreo & & & $\mathbf{4 1 9}$ & $\mathbf{4 2 3}$ & $\mathbf{4 3 2}$ \\
\hline
\end{tabular}

La Foradada y El Mancolibre se contabilizaron un total de 1.274 invertebrados, 419, 423, y 432 en cada isla respectivamente (Tabla 2).

Respecto a los órdenes encontrados, el de Coleoptera (todos ellos, tenebriónidos) fue el más numeroso en todos los casos, alcanzando, en la Illa Grossa, un 88,7 $\%$ del total de los invertebrados contabilizados, seguido de Hemiptera con un 7,6 \% e Isopoda con un 2,3\%.

En El Mancolibre y La Ferrera, el segundo grupo más numeroso fue el de los Isopoda, con un $41,2 \%$ y $10,5 \%$ respectivamente. En esta última isla el siguiente grupo fue el de los escorpiones con un $7.2 \%$, mientras que La Foradada ocurrió al revés, el segundo grupo más numeroso fue los escorpiones seguido muy cerca por los Isopoda, con un $6,6 \%$ y $5,7 \%$ respectivamente.

Por otra parte, se obtuvieron 514 registros entre avistamientos y colectas, de los cuales 443 fueron en La Illa Grossa (40 colectas y 403 avistamientos), 14 en El Mancolibre (todos avistamientos), 31 en La Ferrera ( 2 colectas y 29 avistamientos), 25 en La Foradada (todos avistamientos) y un único avistamiento en La Senyoreta, que correspondía a la especie $L$. italica (Isopoda: Ligiidae).

De todo esto, se han obtenido un total de 45 nuevos taxones de invertebrados continentales para el archipiélago de las Islas Columbretes (Tabla 3 ) (8 arácnidos, 36 insectos y un malacostraco), todas ellas en La Illa Grossa excepto tres, Coccinella septempunctata Linnaeus, 1758 (Coleoptera: Coccinellidae), Ctenolepisma sp. (Zygentoma: Lepismatidae) y Parasteropleurus sp. (Orthoptera: Bradyporidae) en La Ferrera. De las 45 citas, sólo 35 se pudieron identificar hasta especie, el resto, por tratarse de inmaduros o por disponer sólo de fotografías en las que no se apreciaban los caracteres necesarios para su determinación, se identificaron tan solo hasta género. En esta tabla también se muestran sombreadas aquellas especies que se recolectaron en las trampas de caída (con independencia de que algunas de estas también se registraran mediante avistamientos o colectas) y se muestran sin sombrear aquellas especies que únicamente se registraron mediante avistamientos o colectas, siendo estas la gran mayoría. La Tabla 4 muestra los artrópodos citados por primera vez en alguna de las islas o islotes de Columbretes pero que ya habían sido citados en el archipiélago con anterioridad. Se han registrado cuatro taxones nuevos para La Ferrera, cuatro en La Foradada y cinco en El Mancolibre.

\section{Discusión}

Como se ha comentado con anterioridad en este trabajo, pese a haber sido importante la catalogación faunística de este enclave para todos los grupos zoológicos, la revisión de la bibliografía evidenció una escasez o disminución notable en los trabajos centrados en invertebrados en las últimas décadas. Por lo tanto, y aunque somos conscientes que las identificaciones en base a fotografías limitan mucho una catalogación precisa, en los casos en los que solo ha sido posible identificar la muestra hasta el nivel de género se ha decidido reflejar los nuevos registros que aportamos al corresponderse con primeras citas a este nivel en el archipiélago pues consideramos se amplía de manera relevante la información faunística sobre esta característica Reserva Natural.

Como se indica en la Tabla 3, de los 45 taxones nuevos que aportamos en este trabajo, 16 familias y 40 géneros no se habían registrado con anterioridad en este enclave. En esta misma tabla se puede comprobar que la mayor parte de estos nuevos taxones fueron hallados mediante avistamientos o colectas pues, aunque con las trampas de caída se recolectaron la mayor cantidad de individuos, la gran mayoría de especies se correspondieron con taxones que ya estaban citados con anterioridad en el archipiélago. La distribución conocida de cada una de las nuevas especies y géneros registrados refleja que todos están presentes en la costa peninsular más próxima. Todo esto corrobora lo ya expuesto por Español (1958) y Cartagena (2002), que 
Tabla 3.- Artrópodos citados por primera vez en el archipiélago de Columbretes (clase, orden, familia, género y especie). Se proporciona también la distribución del taxón (sólo a nivel de especie), el nombre de la isla o islote en que se ha encontrado, el año del primer registro tomado en este estudio (años de estudio en Illa Grossa: 2004-2013; en La Ferrera: 2006-2010; y en La Foradada: 2008-2010) y frecuencia de observaciones ( $n^{\circ}$ de años en que la especie se ha observado a partir de ese primer registro y, entre paréntesis, número de años transcurridos desde ese primer registro y el final del estudio en la correspondiente isla). IG =Illa Grossa, Frec.=Frecuencia, Cosmop.=Cosmopolita, P.I.=Península lbérica, $S=S u r, S E=S u r e s t e$, $\mathrm{E}=$ Este, Occ. $=$ Occidental, Or.=Oriental, Med. $=$ Mediterráneo, Intro. $=$ Introducción, C.p. $=$ Control de plagas, Orig.=Origen, Endem. $=$ Endemismo, Reg. $=$ Regiones, Temp. $=$ Templada, Trop. $=$ Tropical, Migrad. $=$ Especie Migradora. Las especies que no aparecen sombreadas únicamente fueron localizadas mediante avistamientos o colectas.

Table 3.- Arthropods cited for the first time at the Columbretes archipelago (class, order, family, genus and species). The distribution of each taxa (only at species level) and the name of the island or islet where it was found is also given, the year of the first record taken in this study (years of study in Illa Grossa: 2004-2013, in La Ferrera: 2006-2010, and in La Foradada: 2008-2010) and frequency of observations (number of years in which the species was observed from that first record and, in parentheses, number of years passed since that first record and the end of the study in its corresponding island). IG = Illa Grossa, Frec. = Frequency, Cosmop.=Cosmopolitan, P.I.= Iberian peninsula, S=South, SE=Southeast, E=East, Occ.=Western, Or.=Eastern, Med.=Mediterranean, Intro.=Introduced, C.p. $=$ Pest control, Orig. $=$ Origin, Endem. $=$ Endemic, Reg. $=$ Regions, Temp. $=$ Temperate, Trop. $=$ Tropical, Migrad. $=$ Migratory species. Species unshaded were only located through direct visual observations and random collections.

\begin{tabular}{|c|c|c|c|c|c|c|c|}
\hline Clase & Orden & Familia & Especie & Distribución & $\begin{array}{l}\text { Isla/ } \\
\text { islote }\end{array}$ & $\begin{array}{l}1^{\circ} \\
\text { Registro }\end{array}$ & Frec. \\
\hline \multirow{14}{*}{$\begin{array}{l}\frac{\pi}{0} \\
\frac{0}{2} \\
\frac{5}{U} \\
\frac{\pi}{4}\end{array}$} & \multirow{8}{*}{ 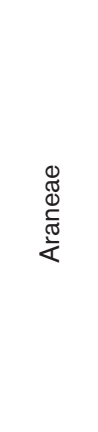 } & Araneidae & Argiope trifasciata (Forskal, 1775) & $\begin{array}{l}\text { Cosmop. P.I, SE de España } \\
\text { y S de Portugal. }\end{array}$ & IG & 2006 & $3(8)$ \\
\hline & & Araneidae & $\begin{array}{l}\text { Neoscona subfusca (C.L. Koch, } \\
\text { 1837) }\end{array}$ & Paleártico Occ. & IG & 2009 & $5(5)$ \\
\hline & & Clubionidae* & Clubiona sp. & & IG & 2009 & $1(5)$ \\
\hline & & Dysderidae & Dysdera cf. crocata ${ }^{*}$ & & IG & 2009 & 1(5) \\
\hline & & Gnaphosidae* & Zelotes sp. & & IG & 2008 & $1(6)$ \\
\hline & & Nemesiidae* & Nemesia sp. & & IG & 2004 & $10(10)$ \\
\hline & & Segestriidae ${ }^{\star}$ & Segestria sp & & IG & 2009 & $1(5)$ \\
\hline & & Therediidae* & Steatoda sp. & & IG & 2008 & $3(6)$ \\
\hline & & Cerambycidae & $\begin{array}{l}\text { Arhopalus syriacus } \\
\text { (Reitter, 1895)* }\end{array}$ & $\begin{array}{l}\text { Med. Or. Intro. accidental en } \\
\text { muchas partes del mundo. }\end{array}$ & IG & 2009 & $1(5)$ \\
\hline & & Coccinellidae* $^{*}$ & $\begin{array}{l}\text { Coccinella septempunctata } \\
\text { Linnaeus, } 1758\end{array}$ & Paleártica. Intro. como C.p. & Ferrera & 2006 & $1(5)$ \\
\hline & $\begin{array}{l}\frac{ \pm}{0} \\
\frac{0}{0}\end{array}$ & Coccinellidae & Rodolia cardinalis (Mulsant, 1850) & $\begin{array}{l}\text { Orig. Australia. Intro. como } \\
\text { C.p. }\end{array}$ & IG & 2004 & $10(10)$ \\
\hline & U & Dynastidae $^{*}$ & $\begin{array}{l}\text { Phyllognathus excavatus (Forster, } \\
\text { 1771) }\end{array}$ & Paleártico Or. E de España. & IG & 2010 & $1(4)$ \\
\hline & & Elateridae $^{\star}$ & $\begin{array}{l}\text { Cebrio superbus Jacquelin |du Val, } \\
1860\end{array}$ & Endem. P.I. S y E de España. & IG & 2006 & $2(8)$ \\
\hline & $\begin{array}{l}\frac{\pi}{\omega} \\
\frac{0}{0} \\
\frac{0}{0} \\
\frac{0}{0}\end{array}$ & Blattidae & $\begin{array}{l}\text { Periplaneta americana (Linnaeus, } \\
\text { 1758) }\end{array}$ & Cosmop. Orig. África. & IG & 2010 & $1(4)$ \\
\hline \multirow{10}{*}{ 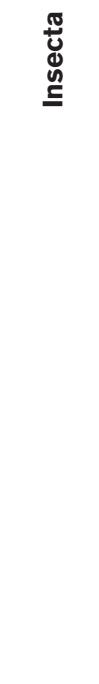 } & $\frac{\pi}{\pi}$ & Calliphoridae & Lucilia sericata (Meigen, 1826) ${ }^{*}$ & Reg. temp. y trop. & IG & 2009 & $5(5)$ \\
\hline & \multirow{6}{*}{ 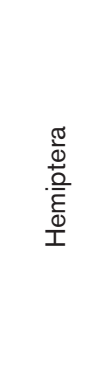 } & Asterolecaniidae ${ }^{*}$ & Planchonia arabidis Signoret, 1876 & Med. & $\mathrm{IG}$ & 2011 & $3(3)$ \\
\hline & & Cydnidae & Cydnus aterrimus (Forster, 1771) & Orig. Med. & IG & 2009 & $5(5)$ \\
\hline & & Margarodidae $^{\star}$ & $\begin{array}{l}\text { Icerya purchasi Maskell, } \\
1878\end{array}$ & $\begin{array}{l}\text { Australia. Intro. accidental en } \\
\text { muchas partes del mundo. }\end{array}$ & IG & 2004 & $10(10)$ \\
\hline & & Pentatomidae & $\begin{array}{l}\text { Brachynema cinctum (Fabricius, } \\
\text { 1775) }\end{array}$ & Paleártica. & IG & 2009 & $1(5)$ \\
\hline & & Pentatomidae & Nezara viridula (Linnaeus, 1758) & Cosmop. & IG & 2009 & $5(5)$ \\
\hline & & Pyrrhocoridae & $\begin{array}{l}\text { Pyrrhocoris apterus (Linnaeus, } \\
1758 \text { ) }\end{array}$ & Paleártica. & IG & 2004 & ( \\
\hline & \multirow{3}{*}{ 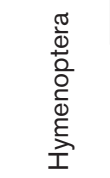 } & Formicidae & Messor bouvieri Bondroit, 1918 & Atlanto-Med & IG & 2010 & 1(4) \\
\hline & & Ichneumonidae & Ophion sp. & & IG & 2010 & $1(4)$ \\
\hline & & Vespidae $^{*}$ & Polistes sp. & & IG & 2009 & $1(5)$ \\
\hline
\end{tabular}


Tabla 3 (continuación).

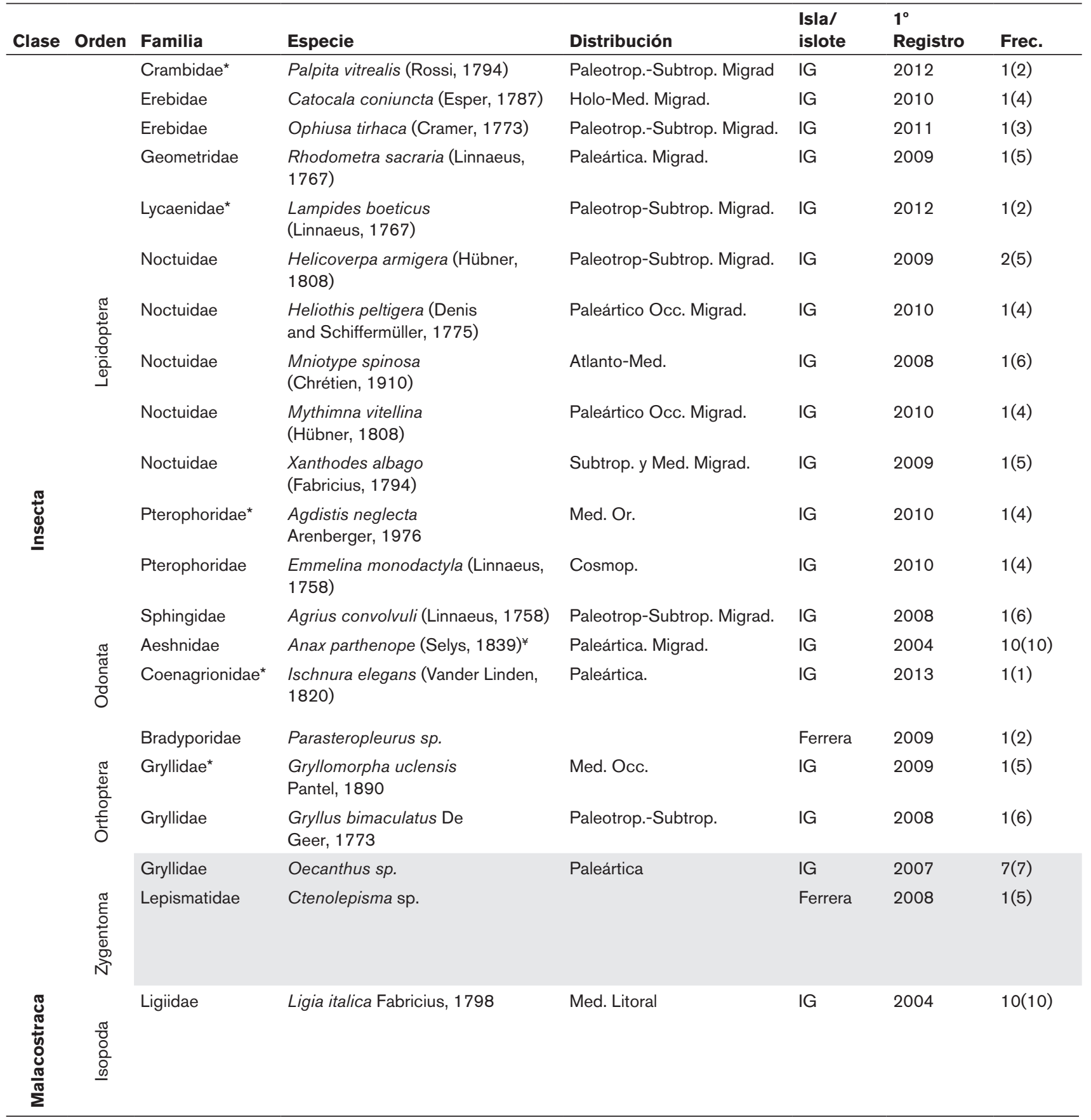

${ }^{*}$ Familias que se citan por primera vez.

$¥$ Géneros que ya habian sido citados con anterioridad.

( D Dato no reflejado porque las frecuencias de las que se disponen hacen referencia a Pyrrhocoridae en general.

establecen que la fauna presente en estas islas tiene que ser similar a la que ocupa las zonas costeras cercanas.

Respecto a la densidad y diversidad de las especies registradas (Tabla 3), lo primero que llama la atención es que, dentro del orden de los Coleoptera, únicamente se recolectaron en las trampas de caída tenebriónidos, siendo el grupo que con más frecuencia y en mayor cantidad fueron recogidos, aunque todas las especies de este grupo ya se habían registrado con anterioridad en La Illa Grossa. Esto era de esperar, pues la utilización de estas trampas constituye una metodología adecuada para la captura de especies de esta familia (Ahearn, 1971; Thomas \& Sleeper, 1977). Los tenebriónidos configuran uno de los grupos mejor adaptados a la colonización de islas y suelen presentar comunidades importantes en todas las del ámbito mediterráneo (Español, 1958). 
Tabla 4.- Artrópodos citados por primera vez en alguna de las islas o islotes de Columbretes pero ya citados en el archipiélago con anterioridad. Año del primer registro tomado en este estudio (años de estudio en La llla Grossa: 2004-2013; en El Mancolibre: 2009-2010; en La Ferrera: 2006-2010; y en La Foradada: 2008-2010) y frecuencia de observaciones ( $n^{\circ}$ de años observado a partir de ese primer registro y, entre paréntesis, número de años transcurridos entre ese primer registro y 2010).

Table 4.- Arthropods cited for the first time in some of the islands or islets of Columbretes but already mentioned in the archipelago before. Year of the first record taken in this study (years of study in Illa Grossa: 2004-2013, in Mancolibre: 20092010, in La Ferrera: 2006-2010, and in La Foradada: 2008-2010) and frequency of observations (number of years observed from that first record and, in parentheses, number of years passed between that first record and 2010).

\begin{tabular}{|c|c|c|c|c|c|c|}
\hline Clase & Orden & Familia & Especie & Isla/islote & $1^{\circ}$ registro & Frec. \\
\hline Arachnida & Scorpiones & Buthidae & Buthus occitanus (Amoreux, 1789) & Mancolibre & 2009 & $1(2)$ \\
\hline \multirow[t]{10}{*}{ Insecta } & Coleoptera & Tenebrionidae & Leptoderis solieri Ferrer, 2015 & Mancolibre & 2009 & $1(2)$ \\
\hline & & Tenebrionidae & Pimelia interjecta Solier, 1836 & Mancolibre & 2009 & $2(2)$ \\
\hline & & Tenebrionidae & Scaurus rugulosus Solier, 1838 & Mancolibre & 2009 & $1(2)$ \\
\hline & & Tenebrionidae & Blaps lusitanica Herbst, 1799 & Ferrera & 2009 & $1(2)$ \\
\hline & & Tenebrionidae & Pimelia interjecta Solier, 1836 & Ferrera & 2008 & $3(3)$ \\
\hline & & Tenebrionidae & Scaurus vicinus Solier, 1838 & Ferrera & 2010 & 1(1) \\
\hline & & Tenebrionidae & Scaurus vicinus Solier, 1838 & Foradada & 2010 & 1(1) \\
\hline & Hemiptera & Pyrrhocoridae* & & Foradada & 2009 & $1(2)$ \\
\hline & Hymenoptera & Formicidae & Messor sp. & Foradada & 2009 & $1(2)$ \\
\hline & Zygentoma & Lepismatidae & Ctenolepisma sp. & Illa Grossa & 2009 & $1(3)$ \\
\hline \multirow[t]{3}{*}{ Malacostraca } & Isopoda & Ligiidae & Ligia italica Fabricius, 1798 & Ferrera & 2006 & $5(5)$ \\
\hline & & Ligiidae & Ligia italica Fabricius, 1798 & Mancolibre & 2009 & $2(2)$ \\
\hline & & Ligiidae & Ligia italica Fabricius, 1798 & Foradada & 2008 & $3(3)$ \\
\hline
\end{tabular}

*Los datos de los que se disponen hacen referencia a Pyrrhocoridae en general.

De todas las especies reflejadas en este estudio, y que no se habían citado con anterioridad en el archipiélago de las Islas Columbretes, sólo una de ellas, Rodolia cardinalis (Mulsant, 1850), es fruto de una introducción intencionada en el archipiélago. Según las Memorias anuales de Gestión e informes internos del personal de la Reserva Natural, desde 2001 se realizan sueltas periódicas de esta especie en La Illa Grossa provenientes del insectario de Almazora (centro dependiente de la Conselleria de Agricultura) para el control biológico del hemíptero margaródido Icerya purchasi Maskell, 1878, pues constituye una plaga que afecta mayoritariamente a la fabácea Medicago citrina, endemismo de área restringida del archipiélago. Según se extrae de dichos documentos, $R$. cardinalis no se ha llegado a establecer en las Columbretes, por lo que cada año se ha precisado sueltas periódicas de este coleóptero.

La totalidad de hemípteros colectados en todas las islas en las trampas de caída corresponde a dos especies de pirrocóridos, Pyrrhocoris apterus (Linnaeus, 1758), especie de hábitos gregarios y totalmente adaptada a una dieta seca compuesta de semillas maduras de plantas del orden Malvales (Socha, 1993) y Scantius aegyptius (Linnaeus, 1758), también asociada a una dieta dependiente de malváceas (Bryant, 2009). Son especies relacionadas con hábitats antrópicos que aprovechan las malvas y lavateras para alimentarse y establecerse. Estas especies de flora son muy abundantes en las Columbretes (Fabregat \&
Laguna, 2013). Pyrrhocoris apterus es una especie nueva para el archipiélago mientras que $S$. aegyptius ya había sido citada con anterioridad (Español, 1958). Pyrrhocoris apterus se ha encontrado ampliamente distribuido por la península ibérica, mientras que $S$. aegyptius se localiza en zonas de la península con influencia mediterránea (Mata et al., 2013).

Respecto al ensífero del género Parasteropleurus localizado en La Ferrera, que corresponde a un estado ninfal, no podemos definir su identidad específica por el momento, puesto que aún están pendientes estudios moleculares y sistemáticos a fin de esclarecer su posición taxonómica. Según los datos preliminares de aquellos análisis, se trataría de una especie con poblaciones en Iberia continental.

Estas especies que damos a conocer por primera vez en el archipiélago han sido en su mayoría especies no frecuentes, observadas un único año durante el estudio (29 de 45, lo que supone el 64\%) mientras que tan sólo unas pocas especies (16 de 45 el 36\% restante) han sido observadas cada año tras su identificación. Entre las especies que solo se han registrado una vez se encuentran taxones migradores. Es en este grupo donde se engloban la mayoría de los nuevos lepidópteros registrados, el coleóptero C. septempunctata, cuyas migraciones suelen ser estacionales (Ricci et al., 2005) y el odonato Anax parthenope (Selys, 1839). Las especies que hemos observado durante todos los años de estudio son: de la clase Arachnida, las arañas Neoscona subfusca (C.L. Koch, 1837) y Nemesia sp.; 
de la clase Insecta, el coleóptero $R$. cardinalis (por el motivo anteriormente expuesto), el díptero Lucilia sericata (Meigen, 1826), los hemípteros Planchonia arabidis Signoret, 1876, Cydnus aterrimus (Forster, 1771), I. purchasi y Nezara viridula (Linnaeus, 1758), el odonato A. parthenope y el ortóptero Oecanthus sp.

Aunque es posible que muchas especies alcanzaran las islas por sus propios medios o sobre objetos a la deriva, para aquellas especies que muestran una predilección manifiesta por hábitats modificados por el ser humano, parece más probable pensar en una introducción accidental desde la península aprovechando el trasiego continuo de materiales, embalajes, contenedores y personal que ha tenido lugar desde la colonización del archipiélago en el siglo XIX, siendo este trasiego en determinadas épocas hasta diario. Podría ser este el caso de Steatoda sp., C. aterrimus, Ctenolepisma sp., Lucilia sp., Gryllus bimaculatus De Geer, 1773 y Arhopalus syriacus (Reitter, 1895), entre otros. En este grupo de especies es reseñable que la ya muy extendida en zonas costeras Periplaneta americana (Linnaeus, 1758), aparece por primera vez en el archipiélago en el año 2010 y no se ha vuelto a registrar desde esa primera observación, por lo tanto, se considera una cita puntual de un ejemplar aislado.

Por el contrario, es relevante la presencia de especies cuyo origen no se explica de manera justificada por la acción, directa o accidental, del ser humano. Es el caso de Cebrio superbus Jacquelin du Val, 1860, de distribución continental localizada. Este género, representado por numerosas especies europeas que ocupan el área mediterránea, no se ha registrado en islas, a excepción de las de mayor tamaño como son Córcega, Cerdeña y Sicilia, y con conocidas conexiones históricas con el continente. Por todo esto, su presencia en las islas Columbretes a lo largo de dos años consecutivos es destacable, ya que las hembras son completamente ápteras y apenas abandonan sus galerías subterráneas. De forma análoga, también es significante la presencia de ejemplares del género Nemesia, de marcado carácter territorial, poco móviles y de comportamientos muy sedentarios en las hembras que, como en el coleóptero anterior, sólo de manera accidental abandonan los nidos en que habitan. De hecho y aunque también está citada en Baleares, el origen de su presencia allí está ligado a las conexiones entre islas en determinados periodos históricos (Mora et al., 2017).

Como era de esperar y debido al diseño del muestreo, la gran mayoría de estas nuevas citas lo son en La Illa Grossa. Aun así, y pese a las inesperadas limitaciones que se nos plantearon en cuanto al diseño experimental ya explicadas en apartados anteriores, cabe destacar la presencia de tres nuevas citas no registradas con anterioridad, en La Ferrera (C. septempunctata y Parasteropleurus sp. y el lepismátido Ctenolepisma sp., detectado este último un año después en La Illa Grossa).
Aunque los islotes más próximos a La Illa Grossa se podrían haber considerado como una única entidad biogeográfica debido a la proximidad con esta, se decidió muestrear por separado El Mancolibre al ser uno de los islotes de mayor entidad del archipiélago, constatar diferencias entre las poblaciones establecidas en ambos hábitats y con el objetivo de obtener unos resultados más completos.

A continuación se especifican las especies que, aún ya citadas en La Illa Grossa con anterioridad, no se habían registrado todavía en alguna de las islas o islotes. Se ha constatado por primera vez la presencia del escorpión B. occitanus y el tenebriónido Scaurus rugulosus Solier, 1838 en El Mancolibre, ambas especies presentes en las otras tres islas. Citamos por primera vez a los tenebriónidos Scaurus vicinus Solier, 1838 en La Ferrera y La Foradada, y Pimelia interjecta Solier, 1836 en El Mancolibre y en La Ferrera. Se ha detectado, también, un registro de hormiga (Messor sp.) determinada, por el momento, sólo hasta género y los pirrocóridos ya citados, en la isla Foradada. Por último, constatamos la presencia de los tenebriónidos Blaps lusitanica Herbst, 1799 en La Ferrera y Leptoderis solieri Ferrer, 2015 en El Mancolibre, especies que tan sólo se habían registrado con anterioridad en La Illa Grossa; aparte de L. italica que, dado que es un animal que ocupa la zona de rompiente y de salpicadura, está adaptado a las condiciones marinas y era esperable su presencia en todas las islas de Columbretes. De estas especies, tan sólo hemos observado durante todos los años de estudio al coleóptero $P$. interjecta y al isópodo L. italica.

Debido a la normativa de la Reserva Natural, nuestra premisa para este estudio fue sacrificar el menor número posible de individuos, pero creemos que habría que tener en cuenta, en futuros estudios, la justificación de recurrir a colectas específicas de determinados grupos cuya identificación precisa de su manipulación en el laboratorio.

\section{Agradecimientos}

A todo el equipo de Columbretes, compañeros y compañeras de trabajo de la Reserva Natural y Marina, por el trabajo de campo (muestreo) y la identificación de algunas especies, especialmente a Valentín Tena Lázaro, Vicente Ferris Malonda, Xavi del Señor de la Gala, Vicente Castañer Franch, Manli Viñes Castro, Honorio Delgado García-Petit, Bruno Sabater Centelles, Kike Sánchez, Raül Ramos García, Sheila Carbó Gironda, Pilar Luis Zaragozà, Manuel Sánchez, Manuel Gómez y Antonio Pérez-Carrión. E. Laguna Lumbreras, P. Pérez Rovira y P. P. Ferrer Gallego. Al departamento de Sanidad Animal (Consellería de Medio Ambiente). A los siguientes especialistas en grupos de invertebrados, por las correspondientes identificaciones y observaciones relativas a su grupo de estudio: Lluc García Socias, Gregorio Ros Montolio, Dra. Ma Ángeles Marcos García, Juan Antonio Zaragoza Miralles, Ferran García-Marí, Eduardo Morano, Santiago Teruel, Daniel García, Dr. Pablo Barranco Vega y Dr. César Monzó. 


\section{Referencias}

Ahearn, G. A., 1971. Ecological factors affecting population sampling of desert tenebrionid beetles. American Midland Naturalist, 86: 385-406. https://doi. org/10.2307/2423632

Alonso, L. A., 1991. Geología estructural del archipiélago de las Columbretes. In: L. A. Alonso, J. L. Carretero \& M. García Carrascosa, M. Islas Columbretes. Contribución al estudio de su medio natural. Consellería d'Administració Pública, Agència del Medi Ambient, Generalitat Valenciana. Valencia: 27-53.

Aparicio, A., Mitjavila, J. M., Araña, V. \& Villa, I. M., 1991. La edad del volcanismo de las islas Columbrete Grande y Alborán (Mediterráneo Occidental). Boletín Geológico y Minero, 102: 74-82.

Bordera, S., 1998. A new species of Fianoniella (Hymenoptera: Ichneumonidae) from the Columbretes Islands (Spain), with additional notes on F. laeviscutum. European Journal of Entomology, 95: 133-140.

Bryant, P. J., 2009. Invasion of Southern California by the Palearctic pyrrhocorid Scantius aegyptius (Hemiptera: Heteroptera: Pyrrhocoridae). The Pan-Pacific Entomologist, 85: 190-193. https://doi.org/10.3956/2009-27.1

Cartagena, M. C., 2002. Medida del estado de conservación de los ecosistemas insulares a través de la utilización de los coleópteros tenebriónidos (Coleoptera, Tenebrionidae). Boletín de la Asociación española de Entomología, 26: 177-192.

Cartagena, M. C. \& Galante, E., 2002. Loss of Iberian island tenebrionid beetles and conservation management recommendations. Journal of Insect Conservation, 6: 73-81.

Castilla, A. M., 1993. Los escorpiones (Buthus occitanus) de Columbretes: abundancia relativa y distribución. In: Estudios de Biología-Parque Natural de las Islas Columbretes. Proyecto PNIC-3-1993. Consellería de Agricultura y Pesca, Generalitat Valenciana. Valencia: 1-3.

Castilla, A. M., Cooper, W. E. \& Pons, G. X., 2010b. The effect of moonlight variation on scorpion (Buthus occitanus) activity in the Columbretes Islands (Mediterranean, Spain). In: V. Pérez-Mellado \& M. M. Ramón. Islands and Evolution. Institut Menorquí d’Estudis, Maó: 221-230.

Castilla, A. M., García, R., Verdugo, I., Pons, G. X. \& Escobar, J. V., 2005. Primeros datos sobre la ecología y comportamiento de las arañas Argiope lobata y A. bruennichi de una población que ha colonizado recientemente las islas Columbretes (Mediterráneo, España). Boletín de la Sociedad de Historia Natural de las Baleares, 48: 61-69.

Castilla, A. M., Herrel, A. \& Gosá, A., 2009. Predation by scorpions (Buthus occitanus) on Podarcis atrata from the Columbretes Islands. Munibe (Ciencias NaturalesNatur Zientziak), 57: 299-302.

Castilla, A. M., Pastor, E. \& Pons, G. X., 2006. Fluctuación anual de la densidad de arañas tejedoras del género Argiope en las islas Columbretes: Consecuencias sobre la lagartija endémica Podarcis atrata. Boletín de la Sociedad de Historia Natural de las Baleares, 49: 137-143.
Castilla, A. M. \& Pons, G. X., 2007. Primeros datos sobre la población de escorpiones (Buthus occitanus) en las islas Columbretes (Mediterráneo, España). Boletín de la Sociedad de Historia Natural de las Baleares, 50: 267-278.

Castilla, A. M., Pons, G. X. \& Escobar, J. V., 2004. Consideraciones ecológicas y biogeográficas del género Argiope (Arachnida, Araneae) en las Islas Columbretes (Castellón, España). Boletín de la Sociedad de Historia Natural de las Baleares, 47: 101-110.

Castilla, A. M., Pons, G. X. \& Gosá, A., 2010a. Seguimiento de una invasión de arañas del género Argiope (Arachnida, Araneae) en las Islas Columbretes. Boletín de la Sociedad de Historia Natural de las Baleares, 53: 123-132.

Castilla, A. M., Vanhooydonck, B. \& Catennazi, A., 2008. Feeding behavior of the Columbretes lizard Podarcis atrata, in relation to Isopoda (Crustaceae) species: Ligia italica and Armadillo officinalis. Belgian Journal of Zoology, 138: 146-148.

Compte, A., 1969. Los coleópteros de las Islas Columbretes. Eos, 45: 97-135.

Domingo, J., Montagud, S. \& Sendra, A., 2007. Invertebrados endémicos de la Comunitat Valenciana. Conselleria de Territori i Habitatge, Generalitat Valenciana. Valencia. 254 pp.

Español, F. 1958. Contribución al conocimiento de los artrópodos y moluscos terrestres de las Islas Columbretes. Miscel-lània Zoològica, 1: 3-37.

Fabregat, C. \& Laguna, E., 2013. Del fuego a la naturaleza: evolución, restauración y conservación de la flora de las Islas Columbretes. In: E. Cardona, I. Estaún, M. Comas, M. \& P. Fraga. Islas y plantas: preservación y comprensión de la flora en las islas mediterráneas. Consell Insular de Menorca. Maó: 297-308.

Gadea, E. 1974. Nematodos liquenícolas de Columbretes. Miscel-lània Zoològica, 3: 4.

García-Marí, F., Ferragut, F., Navarro, V., Laborda, R. \& Costa-Comelles, J., 1991. Nuevas aportaciones al conocimiento de los artrópodos de las Islas Columbretes. In: L.A. Alonso, J.L. Carretero \& M. García Carrascosa. Islas Columbretes. Contribución al estudio de su medio natural. Consellería d'Administració Pública, Agència del Medi Ambient, Generalitat Valenciana. Valencia: 27-53.

Habsburg-Lothringen, Salvator L. von, 1895. Columbretes. Publicacions de l'Excelentíssim Ajuntament de Castelló de la Plana. 1990.

Jaeckel, S., 1952. Die Mollusken der spanischen Mittelmeer-lnseln. Mitteilungen aus dem Zoologisches Museum Berlin, 28: 55-143.

Maillard, A. \& Mauffret, A., 1993. Structure et volcanisme dans la fosse de Valence (Méditerranée Nord-Occidentale). Bulletin de la Société Géologique de France, 164(3): 365-383.

Martínez-Ortí, A. \& Puente, A. I., 2011. Xerocrassa molinae. In: J. R. Verdú, C. Numa \& E. Galante. Atlas y Libro rojo de los Invertebrados amenazados de España (Especies Vulnerables), Vol. II. Dirección General de Medio Natural y Política Forestal, Ministerio de Medio Ambiente, Medio rural y Marino. Madrid: 968-970. 
Mata, L., Grosso-Silva, J. M. \& Goula, M., 2013. Pyrrhocoridae from the Iberian Peninsula (Hemiptera: Heteroptera). Heteropterus Revista de Entomología, 13: 175-189.

Melià, A. 2003. Estudio faunístico de los pulgones (Homoptera: Aphididae) de Castellón. Fundación Caja Castellón-Bancaja: 65-66.

Mestre-Forés, E., 2017. Contribució al coneixement del medi natural i l'empremta humana de la Reserva Natural de les Illes Columbretes: clima, fauna invertebrada $i$ memoria. Tesis doctoral. Universitat Jaume I.

Mora, E., Paspati, A., Decae, A. E. \& Arnedo, M., 2017. Rafting spiders or drifting islands? Origins and diversification of the endemic trap-door spiders from the Balearic Islands, Western Mediterranean. Journal of Biogeography, 44(4): 924-936. https://doi.org/10.1111/ jbi.12885

Muñoz, A., Lastras, G., Ballesteros, M., Canals, M., Acosta, J. \& Uchupi, E., 2005. Sea floor morphology of the Ebro shelf in the region of the Columbretes Islands, western Mediterranean. Geomorphology, 72: 1-18. https://doi.org/10.1016/j.geomorph.2005.04.012

Navarro, P., Escribano, V. \& Lluch, J., 2015. Nematodos liquenícolas de Columbretes. Nematodos Tylenchina del norte de L'Illa Grossa (Reserva Natural de las Islas Columbretes, Comunidad Valenciana). XXI Reunió Biennal de la Reial Societat Espanyola d'Història Natural.

Pérez Bañón, C., 2000. Biología de los sírfidos (Diptera: Syrphidae) de los ecosistemas insulares de la Comunidad Valenciana: aspectos de la relación sírfido-planta. Tesis Doctoral. Universidad de Alicante. Alicante.

Prieto, M. \& Háva, J., 2013. Aportaciones corológicas de la colección del Museu de Ciències Naturals de Barcelona a la fauna iberobalear del género Dermestes Linnaeus, 1758 (Coleoptera, Dermestidae). Arxius de Miscel·lània
Zoològica, 11: 80-116. https://doi.org/10.32800/ amz.2013.11.0080

Ricci, C., Ponti, L. \& Pires, A., 2005. Migratory flight and pre-diapause feeding of Coccinella septempunctata (Coleoptera) adults in agricultural and mountain ecosystems of Central Italy. European Journal of Entomology, 102(3): 531-538. https://doi.org/10.14411/eje.2005.076

Robles, F., 1991. Los gasterópodos terrestres de las Islas Columbretes. In: L. A. Alonso, J. L. Carretero \& M. García Carrascosa. Islas Columbretes. Contribución al estudio de su medio natural. Consellería d'Administració Pública, Agència del Medi Ambient, Generalitat Valenciana, Valencia: 155-A-131-A.

Serrano, R., 1987. Historia de los asentamientos humanos en las Columbretes. In: L. A. Alonso, J. L. Carretero \& M. García Carrascosa. Islas Columbretes. Contribución al estudio de su medio natural. Consellería d'Administració Pública, Agència del Medi Ambient, Generalitat Valenciana, Valencia: 13-18.

Smyth, W. H., 1831. On the Columbretes, volcanic rocks near the coast of Valencia, in Spain. Journal of the Royal Geographical Society of London, 1: 58-62. https://doi. org/10.2307/1797659

Socha, R., 1993. Pyrrhocoris apterus (Heteroptera) - an experimental model species: A review. European Journal of Entomology, 90: 241-286.

Southwood, T. R. E. \& Henderson, P. A., 2000. Ecological Methods. Third Edition. Blackwell Science. $575 \mathrm{pp}$.

Thomas D. B. \& Sleeper, E. L., 1977. The use of pitfall traps for estimating the abundance of arthropods with special reference to the Tenebrionidae (Coleoptera). Annals of the Entomological Society of America 70: 242-248. 